Vesna Degoricija, Matias Trbušić, Ines Potočnjak, Bojana Radulović, Gudrun Pregartner, Andrea Berghold, Hubert Scharnagl, Tatjana Stojakovic, Beate Tiran and Saša Frank*

\title{
Serum concentrations of free fatty acids are associated with 3-month mortality in acute heart failure patients
}

https://doi.org/10.1515/cclm-2019-0037

Received January 11, 2019; accepted May 2, 2019; previously published online June 7, 2019

\section{Abstract}

Background: Plasma free fatty acids (FFA) are higher in heart failure (HF) patients compared to healthy controls. Considering that the extent of FFA elevation in HF might mirror the severity of $\mathrm{HF}$, we hypothesized that the serum levels of FFA may be a useful prognostic indicator for 3-month mortality in acute heart failure (AHF).

Methods: We analyzed the serum samples of AHF patients obtained at admission to the emergency department. Serum levels of FFA were analyzed using an enzymatic reagent on an automatic analyzer.

Results: Out of 152 included AHF patients that were originally included, serum samples of 132 patients were available for the quantification of FFA. Of these, 35 (26.5\%) died within 3 months of onset of AHF. These patients had significantly higher serum levels of FFA compared to AHF patients who were alive 3 months after onset of AHF. Univariable logistic regression analyses showed a significant positive association of FFA levels with 3-month mortality (odds ratio [OR] 2.76 [95\% confidence interval 1.32-6.27],

*Corresponding author: Saša Frank, PhD, Gottfried Schatz Research Center for Cell Signaling, Metabolism and Aging, Molecular Biology and Biochemistry, Medical University of Graz, Neue Stiftingtalstr. 6/6, 8010 Graz, Austria, Phone: +43 31638571969 , Fax:+43316385 79615, E-mail: sasa.frank@medunigraz.at Vesna Degoricija and Matias Trbušić: University of Zagreb School of Medicine, Zagreb, Croatia; and Department of Medicine, University Hospital Centre Sisters of Charity, Zagreb, Croatia

Ines Potočnjak: Department of Medicine, University Hospital Centre Sisters of Charity, Zagreb, Croatia

Bojana Radulović: University Hospital Centre Zagreb, Zagreb, Croatia

Gudrun Pregartner and Andrea Berghold: Institute for Medical Informatics, Statistics and Documentation, Medical University of Graz, Graz, Austria

Hubert Scharnagl, Tatjana Stojakovic and Beate Tiran: Clinical Institute of Medical and Chemical Laboratory Diagnostics, Medical University of Graz, Graz, Austria $\mathrm{p}=0.010$ ). Importantly, this association remained significant after adjusting for age and sex, as well as for further clinical and laboratory parameters that showed a significant association with 3-month mortality in the univariate analyses.

Conclusions: We conclude that the admission serum levels of FFA are associated with 3-month mortality in AHF patients. Therefore, measurements of circulating FFA levels may help identifying high-risk AHF patients.

Keywords: acute heart failure; free fatty acids; lipolysis; mortality.

\section{Introduction}

Heart failure (HF) is a final stage of various cardiovascular diseases and therefore a frequent cause of disability and death worldwide [1].

Hemodynamic impairment in HF triggers compensatory mechanisms causing neurohormonal and metabolic dysfunction, which contribute to the disease progression and severity [2]. Left ventricular dysfunction and heart congestion trigger a compensatory elevation of catecholamines and natriuretic peptides. By contrast, right-sided $\mathrm{HF}$ and the consequent peripheral venous congestion triggers a systemic inflammatory response [3, 4]. Increased serum levels of catecholamines, natriuretic peptides and inflammatory cytokines are the principal drivers of catabolic dominance, in particular adipose tissue lipolysis, the hallmark of metabolic dysfunction in $\operatorname{HF}[5,6]$. Free fatty acids (FFA) generated during lipolysis are transported to the liver, skeletal muscles and the heart, where they are used as an energy substrate. Consequently, the serum levels of FFA are determined by the rate of their generation and usage.

High concentrations of circulating FFA are potent inducers of insulin resistance and systemic metabolic impairment [7]. Elevated FFA have been shown to promote vascular oxidative stress and inflammatory response, 
both the known triggers and hallmark of endothelial dysfunction [8-10]. Together with an augmented myocardial FFA uptake and utilization as energy substrate, as well as excessive FFA storage in cardiomyocites, these may contribute to myocardial dysfunction [11, 12]. Plasma FFA have been found to be higher in HF patients compared to healthy controls [13] and raised in HF patients with reduced rather than preserved left ventricular ejection fraction (EF) [14].

If the extent of FFA elevation in HF mirrors the hemodynamic impairment, then we hypothesized that the serum levels of FFA in the patients' serum at admission may have a prognostic value for mortality in AHF. The aim of the present study was therefore to evaluate the prognostic value of the serum levels of FFA for 3-month mortality in AHF patients.

\section{Materials and methods}

\author{
Study design and patients
}

This was a prospective, observational single-center study in AHF. All subjects gave their informed consent for inclusion prior to participation in the study which was conducted in compliance with the ethical guidelines of the Declaration of Helsinki [15]. Ethics Committee approvals were obtained from the University Hospital Centre Sisters of Charity, Zagreb, Croatia (EP-15389/13-4) and the Medical University of Graz, Austria (29-266 ex 16/17). Patients with AHF as a primary diagnosis were recruited from the Emergency Department between November 2013 and February 2015. The diagnosis of AHF was established according to the ESC and ACCF/AHA Guidelines for HF by time of onset, final clinical presentation and ejection fraction (EF) [16-18]. Left ventricular EF was automatically calculated using the Simpson method. Patients with severe renal failure (serum creatinine $\geq 400 \mu \mathrm{mol} / \mathrm{L}$ ) renal replacement therapy, major systemic disease, severe hepatic cirrhosis (Child-Pugh Class B or C), neoplasms, acute or chronic inflammatory disease at admission, recent trauma or surgery, pregnancy or younger than 18 years were not included in the study. A further exclusion criterion was the patient's choice not to participate in the study. One hundred and fifty-two included AHF patients were treated according to the ESC Guidelines for AHF [17, 18]. Hypertension was diagnosed according to the ESC criteria [19]. Mean arterial pressure (MAP) was calculated as (systolic $+2 \cdot$ diastolic blood pressure)/3. Systolic pulmonary blood pressure (SPAP) was approximated by the tricuspid valve velocity, the estimated central vein pressure and the Bernoulli equation from a Doppler echocardiography. The New York Heart Association (NYHA) Functional Classification is estimated according to the severity of patients' symptoms, and the differentiation of worsening vs. de novo cases was based on the patients' history.

\section{Laboratory procedures}

The collection of the blood samples, and the standard laboratory methods, have already been described in previous reports on our
AHF cohort [20-23]. Blood from the patients was obtained on admission to the hospital. The fasting status of the included AHF patients was unknown. Routine laboratory analyses including serum creatinine, urea, total cholesterol, low-density lipoprotein (LDL) cholesterol, high-density lipoprotein (HDL) cholesterol, triglycerides, alanine aminotransferase (ALT), aspartate aminotransferase (AST), and C-reactive protein (CRP) were measured using a Beckman Coulter instrument AU 2700, 2007 (Brea, CA, USA) and Architect c8000, Abbott 2013 (Chicago, IL, USA). Serum levels of FFA were analyzed using an enzymatic reagent (ACS-ACOD method) from Wako Chemicals (Neuss, Germany) as described previously [24]. The measurements were performed on an Olympus AU640 automatic analyzer. Glomerular filtration rate (GFR) was estimated by the Chronic Kidney Disease Epidemiology Collaboration (CKD-EPI) formula [25]. Interleukin-6 (IL-6) serum levels were determined using a specific chemiluminescent ELISA (QuantiGlo; R\&D Systems, Wiesbaden-Nordenstadt, Germany). An electrochemiluminescence immunoassay with Elecsys e411 (Roche Diagnostics GmbH, Mannheim, Germany) was used for the quantification of $\mathrm{N}$-terminal pro-brain natriuretic peptide (NT-proBNP). Blood cell counts and hemoglobin were measured in full blood supplemented with K EDTA by an automatic analyzer DxH (Beckman Coulter).

\section{Statistical analyses}

FFA serum levels of patients who survived were compared with those who either died in the hospital or within 3 months after onset of AHF using the Mann-Whitney U-test. Correlations between FFA and various laboratory and clinical parameters were determined using Spearman's correlation coefficient due to the skewed distribution of many of the laboratory parameters.

Univariable and multivariable logistic regression analyses were used to examine the impact of FFA on 3-month mortality. In the multivariable analyses, we adjusted for age and sex, as well as for further clinical and laboratory parameters that showed a significant association with 3-month mortality in the univariate analyses. Results are presented as odds ratio (OR) and the respective 95\% CI. R version 3.4.4 was used for these analyses.

\section{Results}

\section{Patients' clinical characteristics and laboratory parameters}

The baseline characteristics, comorbidities, medication, laboratory results and outcome of our 152 AHF patients have been described elsewhere [20-23]. The mean patient age was $75.2 \pm 10.3$ years, with a range of $45.5-96.7$, and 79 $(52 \%)$ were female. Serum samples of 132 patients were available for the analyses presented here. Of these, 35 (26.5\%) died within 3 months of onset of AHF. The median and range for serum FFA levels were 0.87 (0.13-2.71) $\mathrm{mmol} / \mathrm{L}$. There was no difference between AHF patients 
with available FFA measurements and those without, with respect to baseline characteristics, laboratory parameters and comorbidities (Supplementary Tables S1-S3).

\section{Correlation of FFA concentrations with laboratory and clinical parameters}

As shown in Table 1, serum levels of FFA were significantly positively correlated with NT-proBNP, ALT, AST and IL-6. However, the serum levels of FFA were not correlated with age, body mass index (BMI), MAP, glucose and several markers of kidney function. Furthermore, no significant correlation with the serum lipids was found, with the exception of HDL cholesterol, for which a significant negative correlation was observed.

\section{Serum levels of FFA in AHF patients who died within 3 months after onset of AHF were higher compared to those who survived}

Patients who died within 3 months after onset of AHF had significantly higher serum levels of FFA compared

Table 1: Correlation analyses of FFA with clinical and laboratory parameters.

\begin{tabular}{lrrr}
\hline & \multicolumn{2}{c}{ FFA, mmol/L } \\
\cline { 2 - 4 } & $\mathbf{r}$ & p-Value & $\mathbf{n}$ \\
\hline Age, years & 0.08 & 0.360 & 132 \\
BMI, $\mathrm{kg} / \mathrm{m}^{2}$ & -0.12 & 0.185 & 132 \\
MAP, mmHg & -0.04 & 0.685 & 132 \\
NT-proBNP, pg/mL & 0.27 & $\mathbf{0 . 0 0 2}$ & 126 \\
GFR, mL/min/1.73 m² & -0.04 & 0.690 & 131 \\
Urea, mmol/L & 0.14 & 0.111 & 131 \\
Creatinine, mol/L & 0.05 & 0.589 & 131 \\
ALT, U/L & 0.20 & $\mathbf{0 . 0 2 1}$ & 128 \\
AST, U/L & 0.24 & $\mathbf{0 . 0 0 5}$ & 129 \\
IL-6, pg/mL & 0.24 & $\mathbf{0 . 0 0 5}$ & 132 \\
Total cholesterol, mmol/L & -0.17 & 0.057 & 132 \\
LDL cholesterol, mmol/L & -0.13 & 0.126 & 132 \\
HDL cholesterol, mmol/L & -0.18 & $\mathbf{0 . 0 4 3}$ & 132 \\
Triglycerides, mmol/L & -0.05 & 0.542 & 132 \\
Glucose, mmol/L & -0.03 & 0.697 & 127 \\
\hline
\end{tabular}

Data presented are the Spearman correlation coefficient $r$, the corresponding $p$-value, and number of available samples (n); significant correlations are depicted in bold. ALT, alanine aminotransferase; AST, aspartate aminotransferase; BMI, body mass index; FFA, free fatty acids; GFR, glomerular filtration rate; HDL, high-density lipoprotein; IL-6, interleukin 6; LDL, low-density lipoprotein; MAP, mean arterial pressure; NT-proBNP, N-terminal pro brain natriuretic peptide.

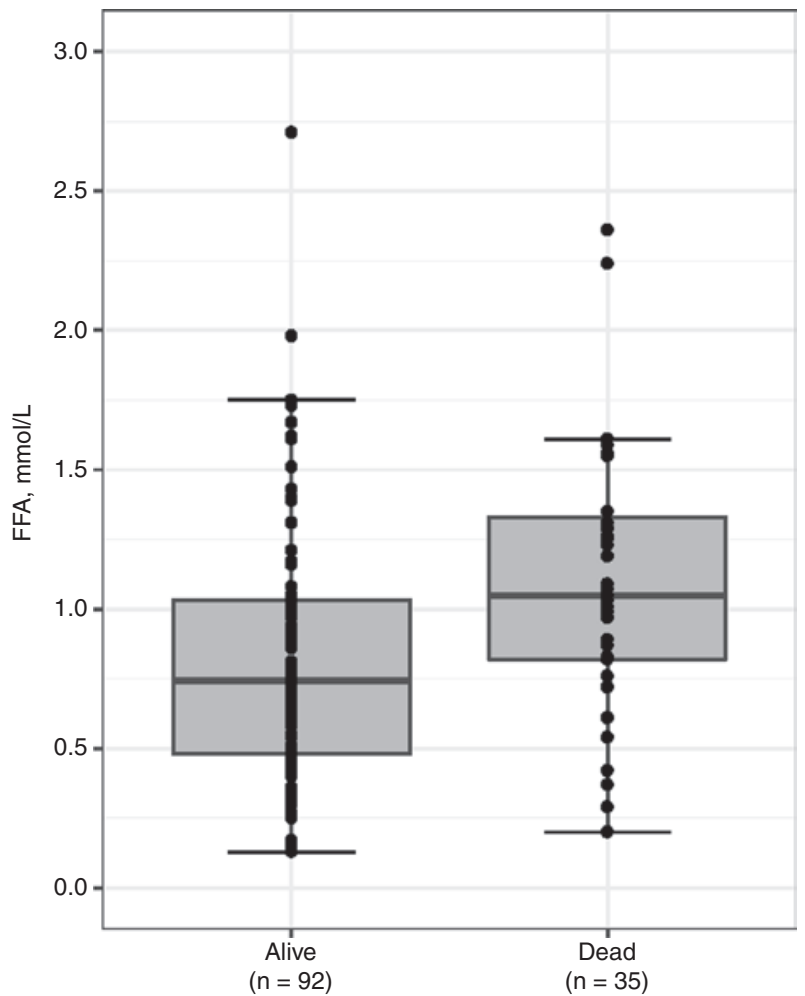

Figure 1: Serum levels of FFA in AHF patients.

Difference between patients who died within 3 months after onset of AHF and those who survived.

to AHF patients who were alive 3 months after onset of AHF (median and range: 0.74 [0.13-2.71] mmol/L vs. 1.05 [0.20-2.36] mmol/L, $\mathrm{p}=0.004$ ) (Figure 1).

\section{Logistic regression analyses}

The univariable analyses showed that 3-month mortality was significantly positively associated with the serum levels of FFA, NT-proBNP, AST, ALT and IL-6, as well as significantly negatively associated with MAP, serum cholesterol levels and GFR (Table 2). Importantly, the association of the serum FFA levels with 3-month mortality remained significant after adjusting for age and sex as well as for the clinical and laboratory parameters which were significantly associated with 3-month mortality in the univariate analyses. Due to the high collinearity between AST and ALT, two different models were fit (Table 3).

\section{Discussion}

This study is the first that shows the association of serum levels of FFA with 3-month mortality in AHF patients. 
Table 2: Univariable logistic regression analyses to assess the association of FFA and various clinical and laboratory parameters with 3-month mortality.

\begin{tabular}{lrrr}
\hline & OR $(\mathbf{9 5} \% \mathrm{Cl})$ & $\mathrm{p}$-Value & Events/n \\
\hline FFA, mmol/L & $2.89(1.30-6.79)$ & $\mathbf{0 . 0 1 0}$ & $35 / 127$ \\
Age, years & $1.02(0.98-1.07)$ & 0.302 & $35 / 127$ \\
Sex & $1.52(0.70-3.37)$ & 0.296 & $35 / 127$ \\
BMI, kg/m² & $0.95(0.88-1.02)$ & 0.185 & $35 / 127$ \\
NT-proBNP, ng/mL & $1.05(1.02-1.09)$ & $\mathbf{0 . 0 0 2}$ & $34 / 121$ \\
GFR, mL/min/1.73 m & $0.97(0.95-0.99)$ & $\mathbf{0 . 0 0 8}$ & $34 / 126$ \\
MAP, mmHg & $0.98(0.96-1.00)$ & $\mathbf{0 . 0 3 0}$ & $35 / 127$ \\
EF, \% & $0.97(0.94-1.01)$ & 0.161 & $27 / 118$ \\
Cholesterol, mmol/L & $0.65(0.40-0.99)$ & $\mathbf{0 . 0 2 9}$ & $35 / 127$ \\
LDL cholesterol, mmol/L & $0.47(0.14-1.36)$ & 0.054 & $35 / 127$ \\
HDL cholesterol, mmol/L & $0.66(0.45-0.94)$ & 0.204 & $35 / 127$ \\
Log(triglycerides), mmol/L & $0.58(0.20-1.54)$ & 0.291 & $35 / 127$ \\
Glucose, mmol/L & $0.94(0.84-1.02)$ & 0.187 & $34 / 123$ \\
AST, U/L & $1.01(1.00-1.02)$ & $\mathbf{0 . 0 1 5}$ & $34 / 125$ \\
ALT, U/L & $1.01(1.00-1.02)$ & $\mathbf{0 . 0 3 1}$ & $34 / 124$ \\
IL-6, pg/mL & $1.01(1.00-1.01)$ & $\mathbf{0 . 0 3 0}$ & $35 / 127$ \\
T2D & $0.93(0.43-2.05)$ & 0.859 & $35 / 126$ \\
Smoking & $0.53(0.18-1.34)$ & 0.202 & $35 / 127$ \\
\hline
\end{tabular}

Significant associations are depicted in bold. ALT, alanine aminotransferase; AST, aspartate aminotransferase; AHF, acute heart failure; $\mathrm{Cl}$, confidence interval; $\mathrm{CRP}, \mathrm{C}$-reactive protein; $\mathrm{EF}$, ejection fraction; FFA, free fatty acids; GFR, glomerular filtration rate; HDL, high-density lipoprotein; IL-6, interleukin-6; LDL, low-density lipoprotein; MAP, mean arterial pressure; NT-proBNP, N-terminal pro brain natriuretic peptide; OR, odds ratio; T2D, type 2 diabetes.

Table 3: Multivariable logistic regression analyses of FFA and 3-month mortality.

\begin{tabular}{lrrr}
\hline & OR $(\mathbf{9 5 \%} \mathrm{Cl})$ & p-Value & Events $/ \mathbf{n}$ \\
\hline Model 1 & $2.94(1.12-8.28)$ & 0.033 & $32 / 118$ \\
Model 2 & $2.77(1.06-7.75)$ & 0.042 & $32 / 117$ \\
\hline
\end{tabular}

Model 1 was adjusted for age, sex, NT-proBNP, GFR, MAP, cholesterol, AST, and IL-6. Model 2 was adjusted for age, sex, NT-proBNP, GFR, MAP, cholesterol, ALT, and IL-6. ALT, alanine aminotransferase; $\mathrm{AST}$, aspartate aminotransferase; $\mathrm{Cl}$, confidence interval; FFA, free fatty acids; GFR, glomerular filtration rate; IL-6, interleukin-6; MAP, mean arterial pressure; NT-proBNP, N-terminal pro brain natriuretic peptide; $\mathrm{OR}$, odds ratio.

After adjusting for variables that were both known to influence the prognosis in HF and significantly associated with 3-month mortality in the univariate analyses in the present study, the association of FFA with 3-month mortality remained significant.

It is known that a decreased cardiac output and a subsequently decreased tissue perfusion as well as venous volume overload and congestion are accompanied by increased serum levels of catecholamines, natriuretic peptides and inflammatory cytokines, which are potent inducers of adipose tissue lipolysis $[5,6,26]$. The positive correlations of serum FFA with NT-proBNP and IL- 6 that were observed in the present study substantiate the relationship between serum levels of FFA, the rate of adipose tissue lipolysis, and the severity of AHF. Therefore, it is reasonable to infer that the higher levels of FFA in AHF patients who died are simply a consequence of the severe stage of the disease itself associated with higher levels of catecholamines and natriuretic peptides as well as a more pronounced inflammatory response and insulin resistance. However, the capacity of elevated circulating FFA to increase the activity of the sympathetic nervous system $[27,28]$ suggests that FFA, which were initially increased as a consequence of hemodynamic and metabolic disturbances in HF, contribute to exaggeration of these pathophysiologic processes. This, together with the established detrimental effects of elevated FFA on the cardiovascular system as well as their role in cardiac lipotoxicity [8-12], suggests that elevated FFA play an active role in the HF pathophysiology. Indeed, previous studies have shown that an increased uptake of FFA into cardiac myocytes, secondary to the elevated FFA serum levels, diminishes glucose utilization and deteriorates cardiac ATP production [29]. In line with this, drugs which interfere with FFA utilization for energy production and shift substrate utilization towards glucose have been shown to exert positive effects on cardiac function in patients with chronic HF [30,31]. Considering this and higher serum FFA levels in AHF patients who died within 3 months after the onset of AHF compared to those who survived, as observed in the present study, it is tempting to assume that the pharmacological inhibition of FFA utilization might improve cardiac function and reduce mortality in AHF.

In the present analysis, serum levels of FFA were also positively correlated with ALT and AST, whose increased serum levels indicate hepatocyte damage. Hepatocyte damage in HF is primarily due to liver hypoperfusion caused by a decreased cardiac output and to a lesser extent due to passive hepatic congestion, a consequence of rightsided $\mathrm{HF}$ [32]. A healthy liver takes up substantial amounts of FFA that are liberated during lipolysis from the adipose tissue and uses them as fuel or substrate for ketone body synthesis. Compared to AHF patients who were alive, AST levels in the present study were significantly higher in AHF patients who died within 3 months after onset of AHF (median and range: 25.0 [10-666] U/L vs. 36.0 [14-487] $\mathrm{U} / \mathrm{L}, \mathrm{p}=0.001$ ). Accordingly, the increased FFA levels in AHF patients who died might at least in part be a consequence of the decreased FFA uptake by the liver, which 
was more damaged in patients who died compared to those that did not.

Additionally, the altered energy substrate preference of the failing heart, namely a shift from FFA to glucose $[5,33]$, may have been more pronounced in patients with a more severe disease. This may have contributed to the higher FFA serum levels in AHF patients who died within 3 months after onset of AHF compared to patients who survived.

There are several limitations to our present study: the design precludes drawing conclusions about cause and effect for the pathophysiological processes involved in the regulation of the serum levels of FFA. Moreover, we have no data on whether and how long patients were fasting before blood collection. Furthermore, due to missing values in the outcome as well as some of the regressor variables, data from only 117 patients was included in the multivariable regression model presented. The moderate number of available serum samples $(n=132)$ in this monocentric study influences the statistical power of our analyses. Therefore, larger studies are needed to confirm our results.

\section{Conclusions}

Based on our results, we conclude that admission serum levels of FFA are associated with 3-month mortality in AHF patients. Therefore, measurements of circulating FFA levels might help identifying high risk AHF patients.

Acknowledgments: The authors thank Sabine Paulitsch and Lusik Balayan for their expert technical assistance.

Conflict of interest: None declared.

Author contributions: Conception and designed by: VD and SF. Acquisition of data: IP, MT, BR, TS, BT and HS. Analysis and interpretation of data: VD, GP, AB and SF. Drafting the manuscript: VD and SF. Revising manuscript for important intellectual content: VD, MT, IP, BR, GP, AB, HS, TS, BT and SF. Final approval of the version to be submitted: VD, MT, IP, BR, GP, AB, HS, TS, BT and SF. All the authors have accepted responsibility for the entire content of this submitted manuscript and approved submission.

Funding: This research was funded by the Austrian Science Fund [P27166-B23 to SF], and the Jubilee Foundation of the Austrian National Bank [15858 to SF].

Employment or leadership: None declared.

Honorarium: None declared.

Competing interests: The funders had no role in the design of the study; in the collection, analyses, or interpretation of data; in the writing of the manuscript, or in the decision to publish the result.

\section{References}

1. Roger VL, Weston SA, Redfield MM, Hellermann-Homan JP, Killian J, Yawn BP, et al. Trends in heart failure incidence and survival in a community-based population. J Am Med Assoc 2004;292:344-50.

2. Packer M. Pathophysiology of chronic heart failure. Lancet 1992;340:88-92.

3. Ambrosy AP, Pang PS, Khan S, Konstam MA, Fonarow GC, Traver B, et al. Clinical course and predictive value of congestion during hospitalization in patients admitted for worsening signs and symptoms of heart failure with reduced ejection fraction: findings from the EVEREST trial. Eur Heart J 2013;34:835-43.

4. Niebauer J, Volk HD, Kemp M, Dominguez M, Schumann RR, Rauchhaus M, et al. Endotoxin and immune activation in chronic heart failure: a prospective cohort study. Lancet 1999;353:1838-42.

5. Doehner W, Frenneaux M, Anker SD. Metabolic impairment in heart failure: the myocardial and systemic perspective. J Am Coll Cardiol 2014;64:1388-400.

6. Szabo T, Postrach E, Mahler A, Kung T, Turhan G, von Haehling S, et al. Increased catabolic activity in adipose tissue of patients with chronic heart failure. Eur J Heart Fail 2013;15:1131-7.

7. Delarue J, Magnan C. Free fatty acids and insulin resistance. Curr Opin Clin Nutr Metab Care 2007;10:142-8.

8. Gambert S, Vergely C, Filomenko R, Moreau D, Bettaieb A, Opie LH, et al. Adverse effects of free fatty acid associated with increased oxidative stress in postischemic isolated rat hearts. Mol Cell Biochem 2006;283:147-52.

9. Steinberg HO, Paradisi G, Hook G, Crowder K, Cronin J, Baron AD. Free fatty acid elevation impairs insulin-mediated vasodilation and nitric oxide production. Diabetes 2000;49:1231-8.

10. Oram JF, Bornfeldt KE. Direct effects of long-chain non-esterified fatty acids on vascular cells and their relevance to macrovascular complications of diabetes. Front Biosci 2004;9:1240-53.

11. Karwi QG, Uddin GM, Ho KL, Lopaschuk GD. Loss of metabolic flexibility in the failing heart. Front Cardiovasc Med 2018;5:68.

12. Opie LH. The metabolic vicious cycle in heart failure. Lancet 2004;364:1733-4.

13. Oie E, Ueland T, Dahl CP, Bohov P, Berge C, Yndestad A, et al. Fatty acid composition in chronic heart failure: low circulating levels of eicosatetraenoic acid and high levels of vaccenic acid are associated with disease severity and mortality. J Intern Med 2011;270:263-72.

14. Zhu N, Jiang W, Wang Y, Wu Y, Chen H, Zhao X. Plasma levels of free fatty acid differ in patients with left ventricular preserved, mid-range, and reduced ejection fraction. BMC Cardiovasc Disord 2018;18:104.

15. World Medical A. World Medical Association Declaration of Helsinki: Ethical Principles for Medical Research Involving Human Subjects. J Am Med Assoc 2013;310:2191-4.

16. Dickstein K, Cohen-Solal A, Filippatos G, McMurray JJ, Ponikowski P, Poole-Wilson PA, et al. ESC guidelines for the diagnosis and treatment of acute and chronic heart failure 2008: the 
Task Force for the diagnosis and treatment of acute and chronic heart failure 2008 of the European Society of Cardiology. Developed in collaboration with the Heart Failure Association of the ESC (HFA) and endorsed by the European Society of Intensive Care Medicine (ESICM). Eur Heart J 2008;29:2388-442.

17. McMurray JJ, Adamopoulos S, Anker SD, Auricchio A, Bohm M, Dickstein K, et al. ESC Guidelines for the diagnosis and treatment of acute and chronic heart failure 2012: the Task Force for the Diagnosis and Treatment of Acute and Chronic Heart Failure 2012 of the European Society of Cardiology. Developed in collaboration with the Heart Failure Association (HFA) of the ESC. Eur Heart J 2012;33:1787-847.

18. Yancy CW, Jessup M, Bozkurt B, Butler J, Casey Jr DE, Drazner MH, et al. 2013 ACCF/AHA guideline for the management of heart failure: a report of the American College of Cardiology Foundation/ American Heart Association Task Force on Practice Guidelines. J Am Coll Cardiol 2013;62:e147-239.

19. Mancia G, De Backer G, Dominiczak A, Cifkova R, Fagard R, Germano G, et al. 2007 Guidelines for the management of arterial hypertension: the Task Force for the Management of Arterial Hypertension of the European Society of Hypertension (ESH) and of the European Society of Cardiology (ESC). J Hypertens 2007;25:1105-87.

20. Radulovic B, Potocnjak I, Dokoza Teresak S, Trbusic M, Vrkic N, Malogorski D, et al. Hypochloraemia as a predictor of developing hyponatraemia and poor outcome in acute heart failure patients. Int J Cardiol 2016;212:237-41.

21. Potocnjak I, Degoricija V, Trbusic M, Teresak SD, Radulovic B, Pregartner G, et al. Metrics of high-density lipoprotein function and hospital mortality in acute heart failure patients. PLoS One 2016;11:e0157507.

22. Potocnjak I, Radulovic B, Degoricija V, Trbusic M, Pregartner G, Berghold A, et al. Serum concentrations of asymmetric and symmetric dimethylarginine are associated with mortality in acute heart failure patients. Int J Cardiol 2018;261:109-13.

23. Degoricija V, Trbusic M, Potocnjak I, Radulovic B, Teresak SD, Pregartner G, et al. Acute heart failure developed as worsening of chronic heart failure is associated with increased mortality compared to de novo cases. Sci Rep 2018;8:9587.
24. Pilz S, Scharnagl H, Tiran B, Wellnitz B, Seelhorst U, Boehm BO, et al. Elevated plasma free fatty acids predict sudden cardiac death: a 6.85-year follow-up of 3315 patients after coronary angiography. Eur Heart J 2007;28:2763-9.

25. Levey AS, Stevens LA, Schmid CH, Zhang YL, Castro 3rd AF, Feldman $\mathrm{HI}$, et al. A new equation to estimate glomerular filtration rate. Ann Intern Med 2009;150:604-12.

26. Lafontan M, Moro C, Berlan M, Crampes F, Sengenes C, Galitzky J. Control of lipolysis by natriuretic peptides and cyclic GMP. Trends Endocrinol Metab 2008;19:130-7.

27. Manzella D, Barbieri M, Rizzo MR, Ragno E, Passariello N, Gambardella A, et al. Role of free fatty acids on cardiac autonomic nervous system in noninsulin-dependent diabetic patients: effects of metabolic control. J Clin Endocrinol Metab 2001;86:2769-74.

28. Stepniakowski KT, Goodfriend TL, Egan BM. Fatty acids enhance vascular alpha-adrenergic sensitivity. Hypertension 1995; 25(4 Pt 2):774-8.

29. Bertero E, Maack C. Metabolic remodelling in heart failure. Nat Rev Cardiol 2018;15:457-70.

30. Lee L, Campbell R, Scheuermann-Freestone M, Taylor R, Gunaruwan P, Williams L, et al. Metabolic modulation with perhexiline in chronic heart failure: a randomized, controlled trial of short-term use of a novel treatment. Circulation 2005;112:3280-8.

31. Zhang L, Lu Y, Jiang H, Zhang L, Sun A, Zou Y, et al. Additional use of trimetazidine in patients with chronic heart failure: a meta-analysis. J Am Coll Cardiol 2012;59:913-22.

32. Laribi S, Mebazaa A. Cardiohepatic syndrome: liver injury in decompensated heart failure. Curr Heart Fail Rep 2014;11:236-40

33. Hunter WG, Kelly JP, McGarrah 3rd RW, Kraus WE, Shah SH Metabolic dysfunction in heart failure: diagnostic, prognostic, and pathophysiologic insights from metabolomic profiling. Curr Heart Fail Rep 2016;13:119-31.

Supplementary Material: The online version of this article offers supplementary material (https://doi.org/10.1515/cclm-2019-0037). 